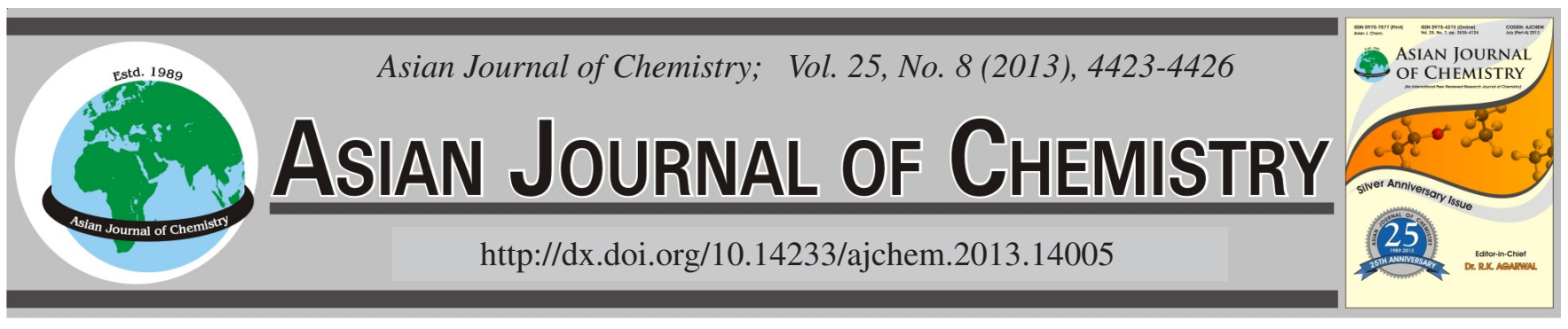

\title{
Synthesis Conditions of Mesoporous NaA Zeolites
}

Ying Meng, Fu-Xiang Li*, Yan-Chao Feng, Jian-Wei Xue and Zhi-Ping Lv

Research Institute of Special Chemicals, Taiyuan University of Technology, Taiyuan 030024, Shanxi Province, P.R. China

*Corresponding author: Fax: +86 351 6111178; Tel: +86 351 6010550-3; Email: L63f64x@163.com

(Received: 3 May 2012;

Accepted: 13 February 2013)

AJC-12980

\begin{abstract}
Mesoporous NaA zeolites were successfully synthesized by a direct hydrothermal method using tetraethylorthosilicate and [3(trimethoxysilyl)propyl]octadecyl-dimethyl ammonium chloride (TPOAC) as the silicon source and mesopore-generating agent, respectively. The effects of the synthesis conditions, including the added amount of [3-(trimethoxysilyl)propyl]octadecyl-dimethyl ammonium chloride, crystallization temperature and gel alkalinity, were investigated. The as-synthesized materials were characterized by X-ray diffraction, nitrogen adsorption-desorption measurements, scanning electron microscopy and transmission electron microscopy. The sample mesopore walls were found to be comprized of NaA zeolite frameworks. The sample size and mesopore structure were affected by the added amount of [3-(trimethoxysilyl)propyl]octadecyl-dimethyl ammonium chloride. The crystal phase was affected by the crystallization temperature and gel alkalinity.
\end{abstract}

Key Words: Mesoporous NaA zeolites, Tetraethylorthosilicate, Synthesis conditions.

\section{INTRODUCTION}

$\mathrm{NaA}$ zeolites are perfect cubic crystals whose cages are connected by eight-atom rings. Owing to the $0.42 \mathrm{~nm}$ effective aperture of $\mathrm{NaA}$ zeolites, they are denoted as $4 \mathrm{~A}$ zeolite molecular sieves. With $70 \% \mathrm{Na}^{+}$exchanged by $\mathrm{Ca}^{2+}$, the effective aperture increases to $0.55 \mathrm{~nm}$ and the obtained zeolites are called $5 \mathrm{~A}$ zeolite molecular sieves. With $70 \% \mathrm{Na}^{+}$ exchanged by $\mathrm{K}^{+}$, the effective aperture is reduced to $0.3 \mathrm{~nm}$ and the acquired zeolites are named $3 \mathrm{~A}$ zeolite molecular sieves. NaA zeolites exhibit many characteristics such as adsorption-selectivity, catalytic-selectivity and ion exchange $e^{1,2}$.

$\mathrm{NaA}$ zeolites act as a kind of adsorbent and desiccant, among others, thus leading to their numerous technical applications in oil refining, petroleum chemical industry, fine chemical industry, medicine, food and light industries, etc. However, the drawback of LTA zeolites is not only the diffusion limitation of larger molecules in the microporous network for catalytic reactions, but also the smaller aperture prevents material transmission in catalytic reactions, which greatly reduce the conversion of the reaction.

To solve the diffusion problem, many researchers have focused on generating mesopores in $\mathrm{NaA}$ zeolites and synthesizing mesoporous $\mathrm{NaA}$ zeolites ${ }^{3,4}$. Mesoporous $\mathrm{NaA}$ zeolites are mesopore-micropore hierarchical zeolites ${ }^{5-15}$. The mesopore walls of these zeolites are composed of microporous crystalline zeolite frameworks, which can not only solve the diffusion problem but also maintain the hydrothermal stability of zeolites. Therefore, they have extensive industrial applications ${ }^{16}$.
Ryoo et al. $^{8}$ reported that mesopores can be generated in $\mathrm{NaA}$ zeolites using TPHAC as the mesopore-directing agent and sodium metasilicate nonahydrate as the silicon source. The present paper reports a direct synthesis route to mesoporous $\mathrm{NaA}$ zeolites using tetraethylorthosilicate and organosilanes \{[3-(trimethoxysilyl)propyl]-octadecyl-dimethyl ammonium chloride, TPOAC $\}$ as the silicon source and mesopore-directing agent, respectively. The effects of the synthesis conditions, including the added amount of TPOAC, crystallization temperature and gel alkalinity, were investigated.

\section{EXPERIMENTAL}

Analytical reagent (AR)-grade sodium aluminate $\left(\mathrm{NaAlO}_{2}\right)$ was purchased from Tianjin Kermel Chemical Reagent Co. Ltd., AR-grade sodium metasilicate nonahydrate $\left(\mathrm{Na}_{2} \mathrm{SiO}_{3} \cdot 9 \mathrm{H}_{2} \mathrm{O}\right)$ was from Tianjin Kermel Chemical Reagent Co. Ltd., ARgrade tetraethylorthosilicate $\left(\mathrm{C}_{8} \mathrm{H}_{20} \mathrm{O}_{4} \mathrm{Si}\right)$ was from Tianjin Damao Chemical Reagent Factory. Distilled $\mathrm{H}_{2} \mathrm{O}$ was made in our laboratory. TPOAC $\left(\mathrm{C}_{26} \mathrm{H}_{58} \mathrm{ClNO}_{3} \mathrm{Si}, 60 \%\right.$ in methanol $)$ was from Gelest, Inc.

Preparation of mesoporous $\mathrm{NaA}$ zeolites: Mesoporous $\mathrm{NaA}$ zeolites were hydrothermally synthesized using TPOAC as the mesopore-directing agent. Typically, a certain amount of sodium aluminate was first dissolved in distilled water. Moderate amounts of tetraethylorthosilicate and TPOAC $(60 \%$ wt $\%$ methanol solution) were then added dropwise to the mixture under vigorous stirring. The obtained mixture was further stirred at room temperature for $1 \mathrm{~d}$ to obtain a homogeneous 
mixture. The synthesis gel was then transferred to a Tefloncoated stainless-steel autoclave and hydrothermally crystallized at $373 \mathrm{~K}$. The precipitated product was cooled, collected by filtering and washed with distilled water until the $\mathrm{pH}$ of the filtrate became neutral. Finally, the product was dried at $373 \mathrm{~K}$ and calcined at $823 \mathrm{~K}$ for $6 \mathrm{~h}$.

Characterization method: Phase composition analysis of the products was performed by X-ray diffraction (XRD) analysis using a Shimadzu X-ray diffractometer LabX XRD-6000 (X-ray beam from nickel-filtered $\mathrm{CuK}_{\alpha}$ radiation, $\lambda=0.15418$ $\mathrm{nm}$, voltage of $40 \mathrm{kV}$, current of $30 \mathrm{~mA}$, scanning rate of $8 \%$ min, step length of $0.02^{\circ}$, divergent and scatter slit size both $1.0^{\circ}$, receiving slit of $0.15 \mathrm{~nm}$ and $2 \theta$ angle interval of $5^{\circ}-35^{\circ}$ ).

The surface area, pore size distribution, pore volume and nitrogen adsorption-desorption isotherms were determined by nitrogen adsorption-desorption measurements on a Micromeritics ASAP 2020. The total surface area was calculated based on the BET method and the pore size distribution in the mesopore range was analyzed by the BJH model using the adsorption branch of isotherms.

The crystal size and morphology of the zeolites were analyzed by field emission scanning electron microscopy (SEM) on a JEOL-JSM-6700F instrument and transmission electron microscopy (TEM) on a JEOL-JEM-1011 microscope.

\section{RESULTS AND DISCUSSION}

Effect of different TPOAC amounts: Fig. 1 shows the XRD patterns of the mesoporous $\mathrm{NaA}$ zeolites synthesized with different TPOAC amounts. When the $n($ TPOAC $) / n\left(\mathrm{SiO}_{2}\right)$ mole ratio is within the range of $0-0.12$, the XRD patterns of the products all exhibit the typical diffraction peak of the $\mathrm{NaA}$ zeolites. However, with increased TPOAC quantity, the diffraction peak widths gradually increase, while the peak intensities decrease. This result can be explained in two ways. First, a higher mole ratio results in smaller crystal sizes, as poined out in Figs. 6a-6c. Second, TPOAC addition led to the formation of mesoporous channels in the NaA zeolites (Fig. 6d), making the crystal imperfect, decreasing peak intensity and increasing the diffraction peak widths. With increased amount of TPOAC, zeolite crystallization requires a longer time. Table-1 presents the time needed for perfect crystal crystallization.

A higher crystallization temperature or synthetic gel alkalinity can reduce the crystallization time and were thus investigated.

Effect of different crystallization temperatures: Fig. 2 shows the XRD patterns of mesoporous NaA zeolites synthesized at different crystallization temperatures for $4 \mathrm{~d}$ at 0.08 $n(\mathrm{TPOAC}) / n\left(\mathrm{SiO}_{2}\right)$ mole ratio. The powder XRD patterns of the product hydrothermally crystallized at $373 \mathrm{~K}$ do not exhibit the typical diffraction peaks of $\mathrm{NaA}$ zeolites, whereas those crystallized at $383 \mathrm{~K}$ have well-resolved peaks resembling those of $\mathrm{NaA}$ zeolites. However, at the crystallization temperature of $393 \mathrm{~K}$, the typical diffraction peaks of sodalite appear, although those of the $\mathrm{NaA}$ zeolites remain. The $\mathrm{NaA}$ zeolites turned into sodalite above $403 \mathrm{~K}$. Hence, increasing the temperature can reduce the crystallization time and improve the crystallinity, but very high temperatures generate sodalite instead of $\mathrm{NaA}$ zeolites.

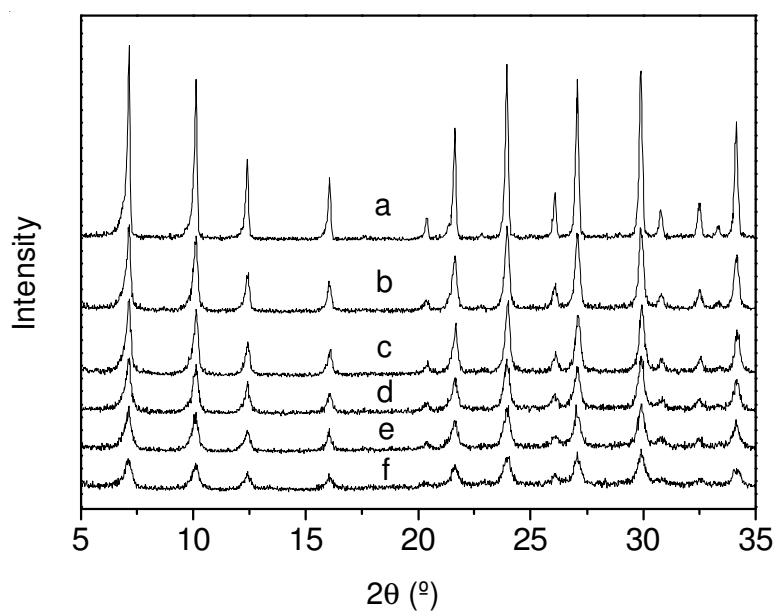

Fig. 1. XRD patterns of mesoporous NaA zeolites synthesized with different amounts of TPOAC. $n($ TPOAC $) / n\left(\mathrm{SiO}_{2}\right)$ : a) 0, b) 0.04 , c) 0.06 , d) 0.08 , e) 0.10, f) 0.12

\begin{tabular}{lcc}
\multicolumn{2}{c}{ TABLE-1 } \\
& \multicolumn{2}{c}{$\begin{array}{c}\text { NaA ZEOLITES CRYSTALLIZATION TIME } \\
\text { WITH VARIOUS AMOUNTS OF TPOAC }\end{array}$} \\
\hline Sample & $n(\mathrm{TPOAC}) / n\left(\mathrm{SiO}_{2}\right)$ & Time $(\mathrm{d})$ \\
\hline $\mathrm{a}, \mathrm{b}$ and c & 0.06 & 4 \\
$\mathrm{~d}$ & 0.08 & 6 \\
$\mathrm{e}$ & 0.10 & 10 \\
$\mathrm{f}$ & 0.12 & 15 \\
\hline
\end{tabular}

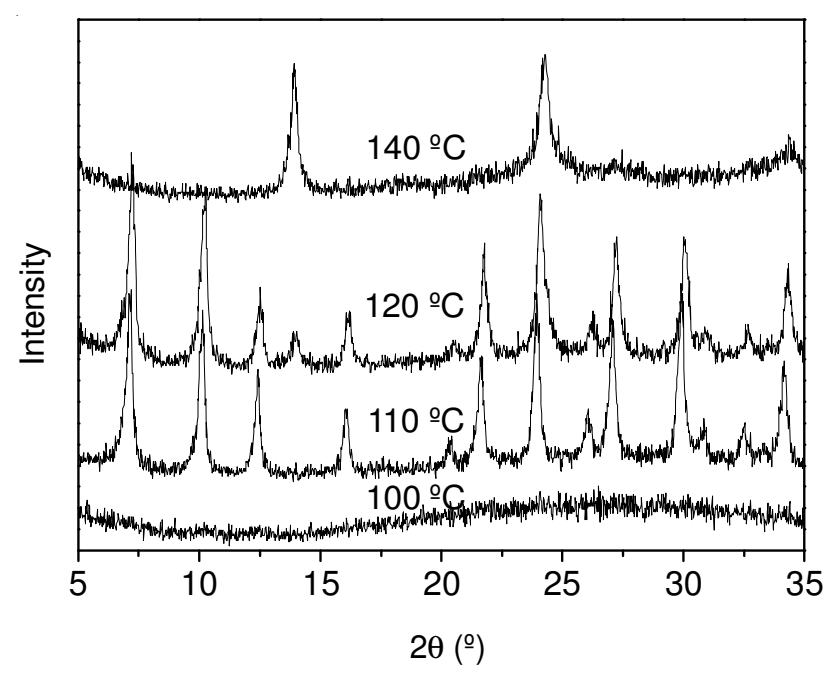

Fig. 2. XRD patterns of mesoporous NaA zeolites synthesized at different crystallization temperatures

Effect of different alkalinity conditions: Fig. 3 shows the XRD patterns of mesoporous $\mathrm{NaA}$ zeolites synthesized at the same $n$ (TPOAC) $/ n\left(\mathrm{SiO}_{2}\right)$ mole ratio $(0.08)$ and at $373 \mathrm{~K}$, but under different alkalinity conditions. When the $n\left(\mathrm{Na}_{2} \mathrm{O}\right)$ / $n\left(\mathrm{SiO}_{2}\right)$ mole ratio is 1.01 or 3.83 , the typical diffraction peaks belonging to $\mathrm{NaA}$ zeolites are absent. When the ratio is 5.24 , the powder XRD patterns of the product exhibit the resolved peaks of NaA zeolites. However, the alkalinity continues to increase, once the ratio reaches 6.65 and the characteristic diffraction peaks of sodalite emerge at $2 \theta=13.9^{\circ}$. The NaA zeolites completely transfrom into sodalite at the ratio of 8.06. Thus, the crystallization time can be cut down by increaseing the alkalinity, but very high alkalinity results in sodalite rather than $\mathrm{NaA}$ zeolites. 


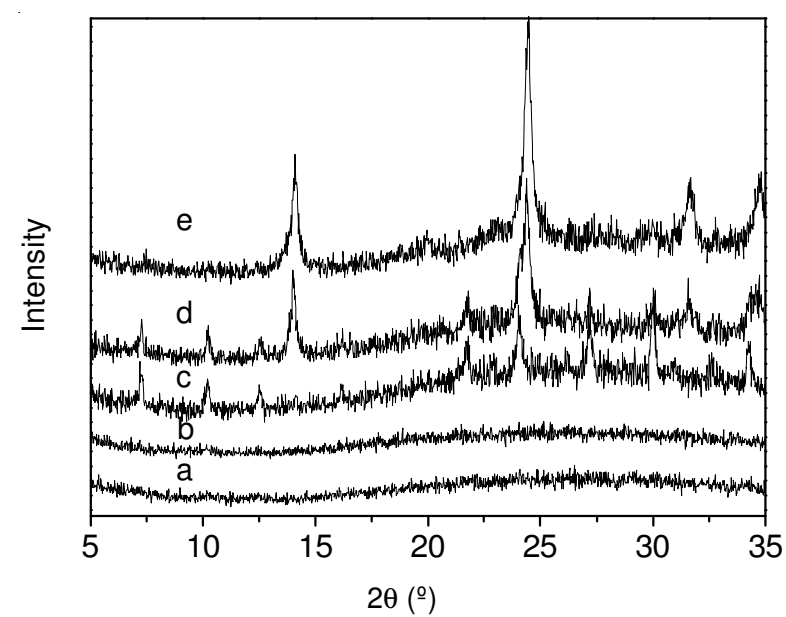

Fig. 3. XRD patterns of mesoporous $\mathrm{NaA}$ zeolites synthesized at different alkalinity conditions. $n\left(\mathrm{Na}_{2} \mathrm{O}\right) / n\left(\mathrm{SiO}_{2}\right)$ : a) 1.01 , b) 3.83 , c) 5.24 , d) 6.65 , e) 8.06

Characterization of the mesoporous textural properties: Fig. 4 shows the nitrogen adsorption-desorption isotherms of mesoporous $\mathrm{NaA}$ zeolites synthesized with various amounts of TPOAC. When the $n$ (TPOAC) $/ n\left(\mathrm{SiO}_{2}\right)$ mole ratio is within the range of 0.04-0.12, the isotherms exhibit a type IV hysteresis loop. This finding indicates that the products possess a characteristic feature of mesoporous materials. The adsorption quantity steeply increases within the ratio range of $0.6-1.0$, which results from the capillary condensation of $\mathrm{N}_{2}$ gas in mesopores. A higher ratio results in a more backward position of the hysteresis loop, which can be attributed to the higher pressure required for capillary condensation due to the incessant enlargement of the mesoporous pore size with increased amount of TPOAC. Fig. 5 shows the corresponding pore diameter distribution curves of the mesoporous $\mathrm{NaA}$ zeolites. The mesoporous pore sizes gradually increase with increased amount of organosilane. This can be attributed to the limited amount of TPOAC that can bond to the NaA zeolite framework surface. Once the amount of TPOAC exceeds the maximum, excess TPOAC becomes a pore-expanding agent by polymerization instead of bonding to the $\mathrm{NaA}$ zeolite framework surface.

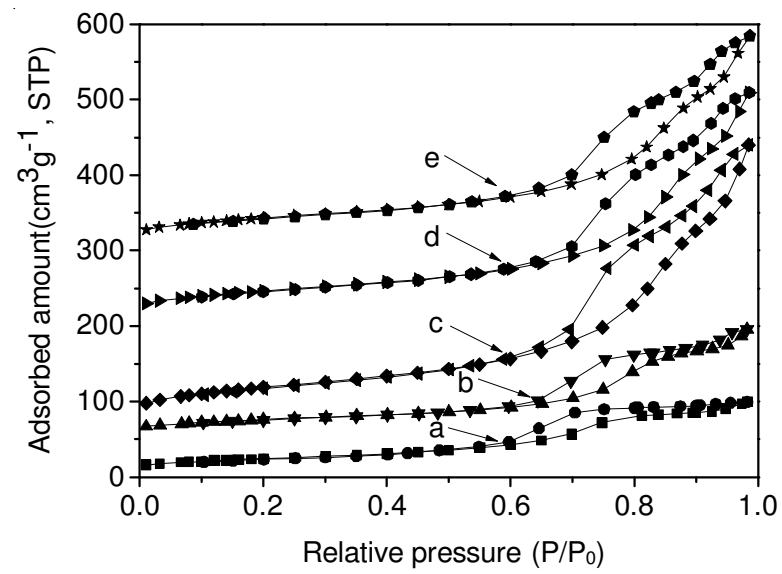

Fig. 4. Nitrogen adsorption-desorption isotherms of mesoporous NaA zeolites synthesized with different amounts of TPOAC. The isotherms for b, c, d and e are vertically offset by 50,60, 200 and $300 \mathrm{~cm}^{3} \mathrm{~g}^{-1}$, respectively. $n(\mathrm{TPOAC}) / n\left(\mathrm{SiO}_{2}\right):$ a) 0.04 , b) $\left.0.06 \mathrm{c}\right) 0.08$, d) 0.10, e) 0.12

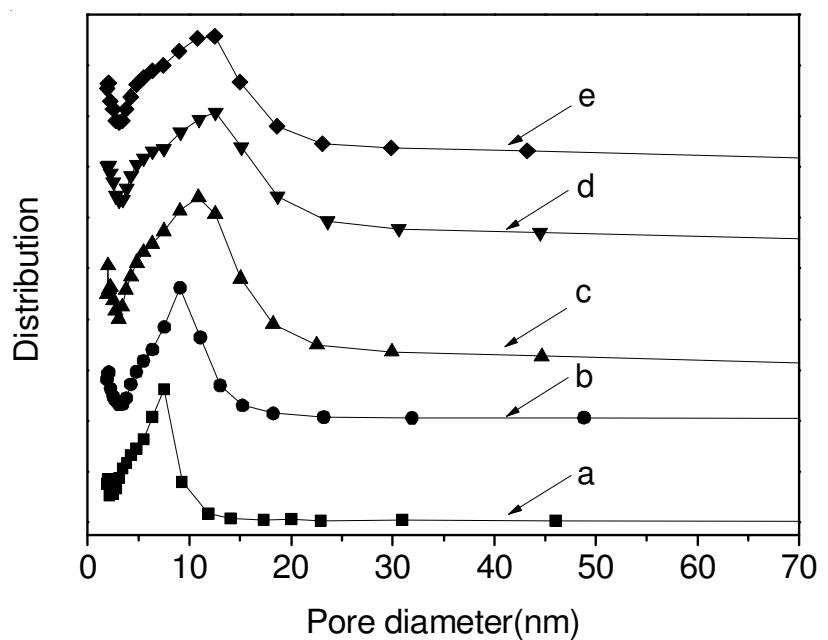

Fig. 5. Pore diameter distribution curves of mesoporous NaA zeolites synthesized with different amounts of TPOAC. $n($ TPOAC $) / n\left(\mathrm{SiO}_{2}\right)$ : a) 0.04 , b) 0.06 c) 0.08 , d) 0.10 , e) 0.12

The pore textural properties of $\mathrm{NaA}$ zeolites synthesized with various amounts of TPOAC are represented in Table-2. With increased $n$ (TPOAC) $/ n\left(\mathrm{SiO}_{2}\right)$ mole ratio, the mesopore volume and BET surface area initially gradually increases and then decreases. At the ratio of 0.08 , the mesopore volume reaches $0.59 \mathrm{~cm}^{3} / \mathrm{g}$ and the BET surface area reaches $212 \mathrm{~m}^{2} / \mathrm{g}$, exceeding that $\left(155 \mathrm{~m}^{2} / \mathrm{g}\right)$ of the product synthesized using TPHAC according to Ryoo et al. ${ }^{4}$. This phenomenon may be due to the mesopore-generating mechanism of the organosilane surfactant. For TPOAC in the synthetic gel, one part is used in bonding to the zeolite framework surface and the other part fills and expands the mesopores. Therefore, with the ratio scaling up within the range of 0.04-0.08, the mesopore volume and BET surface area correspondingly increase. Once the ratio exceeds 0.08 , the concentration of TPOAC in the synthetic gel becomes too heavy that the majority of TPOAC polymerizes with one another. Consequently, the mesopore volume and BET surface area decrease.

\section{TABLE-2}

PORE TEXTURAL PROPERTIES OF NaA ZEOLITES SYNTHESIZED WITH VARIOUS AMOUNTS OF TPOAC

\begin{tabular}{ccccc}
\hline Sample & $n(\mathrm{TPOAC}) / n\left(\mathrm{SiO}_{2}\right)$ & $\mathrm{S}_{\mathrm{BET}}\left(\mathrm{m}^{2} / \mathrm{g}\right)$ & $\mathrm{V}_{\text {mes }}\left(\mathrm{cm}^{3} / \mathrm{g}\right)$ & $\mathrm{D}_{\text {meso }}(\mathrm{nm})$ \\
\hline $\mathrm{a}$ & 0.04 & 88 & 0.15 & 7.6 \\
$\mathrm{~b}$ & 0.06 & 94 & 0.23 & 9.0 \\
$\mathrm{c}$ & 0.08 & 212 & 0.59 & 11.1 \\
$\mathrm{~d}$ & 0.10 & 168 & 0.48 & 12.4 \\
$\mathrm{e}$ & 0.12 & 156 & 0.44 & 11.9 \\
\hline
\end{tabular}

Thus, a higher amount of TPOAC does not result in better pore textural properties of zeolites and the optimal ratio is 0.08 .

Characterization of SEM and TEM: Fig. 6a-6c show the SEM images and Fig. 6d shows the TEM image of mesoporous NaA zeolites synthesized with different amounts of TPOAC. The morphology of the NaA zeolites possesses typical features, including perfect cubic crystal, truncated edges and smooth surfaces. The crystal size ranges typically from $2 \mu \mathrm{m}$ to $3 \mu \mathrm{m}$. The added amount of TPOAC makes the surfaces rough and the truncated edges unclear, which can be attributed to the mesopores existing in the crystal. However, the crystal 
size decreases with increased amount of TPOAC and the cubic crystal turns into a spherical crystal, although the cubic crystalline structure is still maintained. The mesopores existing in the crystal shown by the TEM image prove that the sample is a mesoporous material with $\mathrm{NaA}$ zeolites walls.

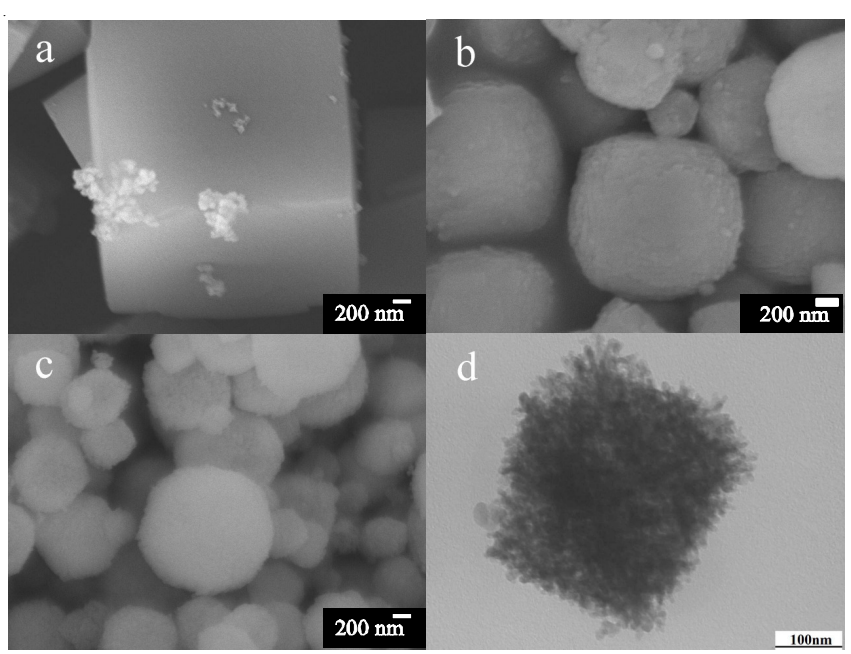

Fig. 6. SEM and TEM images of mesoporous NaA zeolites synthesized with different amounts of TPOAC. $\left.n(\mathrm{TPOAC}) / n\left(\mathrm{SiO}_{2}\right): \mathrm{a}\right) 0$, b) 0.06 , c) 0.10, d) 0.08

\section{Conclusion}

The synthesis of mesoporous $\mathrm{NaA}$ zeolites is strongly influenced by the added amount of TPOAC, crystallization temperature and the gel alkalinity. At the ratio of 0.08 , the mesopore volume and BET surface area both reach the maximum, $0.59 \mathrm{~cm}^{3} / \mathrm{g}$ and $212 \mathrm{~m}^{2} / \mathrm{g}$, respectively. Excess TPOAC can not improve the mesoporous properties and require more time for crystallization. The crystallization time can be decreased by increasing the alkalinity and temperature, but very much high alkalinity and temperature generates sodalite instead of $\mathrm{NaA}$ zeolites. Mesopores exist in the $\mathrm{NaA}$ zeolites, indicating that the sample mesopore walls are comprised of NaA zeolite frameworks.

\section{ACKNOWLEDGEMENTS}

This work was sponsored by the National Natural Science Foundation of China (no. 50972097).

\section{REFERENCES}

1. P.B. Weisz, V.J. Frilette, R.W. Maatman and E.B. Mower, J. Catal., 1, 307 (1962)

2. I. Rosso, C. Galletti, G. Saracco, E. Garrone and V. Specchia, Appl. Catal. B: Environ., 48, 195 (2004).

3. K. Cho, H.S. Cho, L.-C. de Ménorval and R. Ryoo, Chem. Mater, 21, 5664 (2009).

4. S. Tanaka, H. Okada, N. Nakatani, T. Maruo, N. Nishiyama and Y. Miyake, J. Colloid Interf. Sci., 333, 491 (2009).

5. M. Choi, H.S. Cho, R. Srivastava, C. Venkatesan, D. Choi and R. Ryoo, Nat. Mater, 5, 718 (2006).

6. G.V. Shanbhag, M. Choi, J. Kim and R. Ryoo, J. Catal., 264, 88 (2009).

7. X.F. Li, R. Prins and J.A. van Bokhoven, J. Catal., 262, 257 (2009).

8. M. Choi, R. Srivastava and R. Ryoo, Chem. Commun., 42, 4380 (2006).

9. W. Fan, M. A. Snyder, S. Kumar, P. Lee, W. C. Yoo, A. V. Mccormick, R. L. Penn, A. Stein and M. Tsapatsis, Nat. Mater, 7, 984 (2008).

10. D.-H. Lee, M. Choi, B.-W. Yu and and R. Ryoo, Chem. Commun., 74 (2009).

11. Z.L. Zhao, Y.M. Liu, H.H. Wu, X.H. Li, M.Y. He and P. Wu, Micropor. Mesopor. Mater., 123, 324 (2009).

12. K. Suzuki, Y. Aoyagi, N. Katada, M. Choi, R. Ryoo and M. Niwa, Catal. Today, 132, 38 (2008).

13. K.T. Leth, A.K. Rovik, M.S. Holm, M. Brorson, H.J. Jakobsen, J. Skibsted and C.H. Christensen, Appl. Catal. A, 348, 257 (2008).

14. F.-S. Xiao, L.F. Wang, Ch. Y. Yin, K.F. Lin, Y. Di, J.X. Li, R.R. Xu, D. Sh. Su, R. Schlögl, T. Yokoi and T. Tatsumi, Angew. Chem. Int. Ed., 45, 3090 (2006).

15. N. Danilina, F. Krumeich and J.A. van Bokhoven, J. Catal., 272, 37 (2010).

16. Y.S. Tao, H. Kanoh, L. Abrams and K. Kaneko, Chem. Rev., 106, 896 (2006). 